\title{
Klimasensitivität, Leben und die Grenzen der Science-Kultur

\author{
Zum Vierten IPCC-Sachstandsbericht
}

\begin{abstract}
Das IPCC hat mit seinem jüngsten Bericht eine Alarmstimmung ausgelöst. Unter anderem erhöhte es die „beste Schätzung“ der Klimasensitivität (Temperaturerhöhung bei Verdoppelung der $\mathrm{CO}_{2}$-Konzentration) von $2,5^{\circ} \mathrm{C}$ auf $3^{\circ} \mathrm{C}$. Ist diese Korrektur der Beginn einer Tendenz? Das Klimaproblem drängt die Science-Kultur, ihre

Grenzen zu überschreiten und das Klimasystem als Teil des Klima-Erdsystems zu fassen, wobei zunehmend Lebensphänomene zu berücksichtigen sind. Diese dürften die Sensitivität des Systems besonders beeinflussen.
\end{abstract}

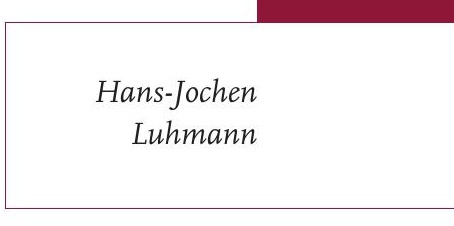

Climate Sensitivity, Life, and the Limits of Science - The IPCC's Fourth Assessment Report | GAIA 17/1 (2008): 25-30 Keywords: certainty, climate change, climate sensitivity, Intergovernmental Panel on Climate Change, IPCC, science, security

\section{Auffällige Betonung des Science-Anspruchs}

Der Vierte Sachstandsbericht (Fourth Assessment Report, AR4) des Intergovernmental Panel on Climate Change (IPCC) ist 2007 erschienen: zuerst der Bericht der Arbeitsgruppe I (WG1) mit dem Titel Climate Change 2007: The Physical Science Basis (IPCC Working Group I 2007), dann die Berichte der beiden weiteren Arbeitsgruppen. Ihre Titel: Climate Change 2007: Impacts, Adaptation and Vulnerability (IPCC Working Group II 2007) und Climate Change 2007: Mitigation of Climate Change (IPCC Working Group III 2007).

Bei den drei Titeln springt eine Asymmetrie ins Auge: Die Titel der Berichte der WG2 und WG3 formulieren das Thematisierte, im Titel des Berichts der WG1 dagegen wird der wissenschaftliche Status des Thematisierten, physical science, mitbenannt. Diese Wendung ist auffällig, denn es ist unstreitig, dass jede IPCC-Arbeitsgruppe wissenschaftliches Wissen zusammenträgt, bedarf also keiner Hervorhebung. Physical science formuliert eine Einschränkung des Verständnisses von „Wissenschaft“ (in vollem Umfang).

In dieser asymmetrischen Titel-Formulierung drückt sich eine Programmatik der Ausgrenzung aus - ob bewusst oder unbewusst. Diese ist angelegt im geläufigen Verständnis „guter Naturwissenschaft" beziehungsweise (neuzeitlicher) Science, das nur rekonstruierbare als „objektive“ Phänomenbeziehungen anerkennt. Ich exemplifiziere an zwei Punkten, wie Science mit

Kontakt: Dr. Hans-Jochen Luhmann | Wuppertal Institut für Klima, Umwelt, Energie | Postfach 100480 | 42004 Wuppertal | Deutschland | Tel.: + 492022492-133 |

E-Mail: jochen.luhmann@wupperinst.org ihrer Programmatik in Konflikt kommen muss angesichts der beim Klimawandel anstehenden Aufgabe:

1. Science basiert auf (intersubjektiv) „sicherer“, unbezweifelbarer Erfahrung: Sie hat das Experiment zum Maßstab der Anerkennung von Erfahrung gemacht. Die Erde, das Erdsystem, aber ist einzig. Von ihr/ihm als Ganze(m) ist experimentelle Erfahrung nicht möglich, auch weil statistische Erfahrung ausgeschlossen ist. Das Erdsystem befindet sich zudem gegenwärtig in einem no-analogue state ${ }^{1}$ : Damit ist auch der Ansatz, wissenschaftliche Erfahrung durch (historische) Analogieschlüsse zu destillieren, erschwert. Science, die einem so speziellen Gegenstand wie dem Erdklima gerecht werden will, muss sich deshalb in ihrem Verständnis von Erfahrung öffnen für die Charakteristika des Klima-Erdsystems und zugleich für die Aufgabe der Mitwahrnehmung des Subjekts.

2. Science nimmt Phänomene nur wahr, sofern sie als Verhalten materieller Körper nach den Gesetzen der Kausalität (in Raum und Zeit) aufzufassen und in diesem Sinne „objektiv“ sind (nach Heisenberg 1943/1944). Auch Phänomene des Lebens unterwirft sie dieser methodischen Maxime und blendet dadurch Wesentliches an ihnen aus. Die Erscheinungen des physikalisch beschreibbaren Klimasystems treten aber innerhalb der Lebenssphäre der Erde auf und sind gegen diese nicht (scharf) abgrenzbar, sondern interferieren vielmehr mit ihr; der Objektivierungsanspruch ist deshalb prekär. Einer Wissenschaft vom Klimasystem, die diesem gerecht werden will, ist es daher verwehrt, Lebensphänomene über diesen Anspruch auszugrenzen.

\footnotetext{
1 So die Formulierung in der Erklärung von Amsterdam von 2001. www.sciconf.igbp.kva.se/ams_declaration.pdf (abgerufen 04.02.2008).
} 


\section{Zwei unscheinbare Auslöser für eine „erhöhte Alarmstufe“}

Die Botschaft des letztjährigen IPCC-Berichts kreist um den Begriff der Sicherheit - in seiner Doppelbedeutung von Gewissheit (Subjekt) und von Unversehrtheit (Objekt). Die Feststellungen des IPCC sind erstens eine größere Sicherheit bezüglich alarmierender Aussagen ${ }^{2}$ und zweitens eine Steigerung des alarmierenden Niveaus. Zum Thema gemacht wird im Weiteren lediglich letzterer Punkt.

Anlass für Alarm und auch dessen Steigerung kann sein, was noch in der offenen Zukunft liegt, etwa Tendenzen des menschlichen Emissionsverhaltens. Anlässe können jedoch auch aus dem Wissen um Fakten und aus strukturellem (zeitmodusinvariantem) Wissen kommen. Es gibt zwei Auslöser für die seitens des IPCC konstatierte erhöhte Alarmstufe:

1. eine Änderung des Standes der Einsicht in die Folgen emittierter Treibhausgase (THG). Der Faktor, der es erlaubt, Ursachen in Folgen, das heißt Temperaturänderung, umzurechnen, heißt „Klimasensitivität“. Die Folgen berücksichtigen sowohl die THG-Emissionen, die der Mensch bereits ausgelöst hat und die sich in Form einer erhöhten Temperatur in Zukunft manifestieren, als auch die Emissionen, die der Mensch in Zukunft noch ausstoßen wird. Die „beste Schätzung “ der Klimasensitivität hat die WG1 des IPCC jetzt von bislang $2,5^{\circ} \mathrm{C}$ auf $3{ }^{\circ} \mathrm{C}$ heraufgesetzt (Abbildung 1$) .^{3}$

2. eine Angabe erhöhter faktischer THG-Emissionen nahe der Gegenwart: a) gegenüber den faktischen Emissionen, als der Dritte IPCC-Sachstandsbericht (Third Assessment Report, TAR) im Jahr 2001 veröffentlicht wurde; b) gegenüber den Angaben der „Science“-Arbeitsgruppe WG1 im AR4 zu den Emissionen am aktuellen Rand. WG1 hat die aktuellen anthropogenen Emissionen nur als Durchschnittswerte mit großer zeitlicher Spannweite und diejenigen aus biogenen Quellen unvollständig angegeben. WG3, die sozialwissenschaftliche Arbeitsgruppe, bietet erstmals Zahlen für die globalen anthropogenen THG-Emissionen für das Jahr $2004^{4}$ sowie bei biogenen Emissionen unter Einschluss solcher aus Moordegradation $^{5}$; c) gegenüber den Schätzwerten für das Jahr 2004, die in den IPCC-Szenarien verwendet wurden. ${ }^{6}$ Diese Schätzwerte, die in den im AR4 berücksichtigten wissenschaftlichen Arbeiten unterstellt sind, liegen in der Gegenwart um etwa fünf Gigatonnen pro Jahr zu tief. Die lange Zeitdauer, die die Arbeitsweise des IPCC erzwingt, hat zur Folge: Der aktuelle Emissionsstand (2004) konnte für die Berechnung der zulässigen künftigen THG-Emissionsbudgets, sollen gewisse Temperaturhöchstwerte nicht überschritten werden, nur mit der Unterschätzung um fünf Gigatonnen pro Jahr Eingang finden.

Es sind diese beiden Gründe, die die Steigerung der Alarmstufe rechtfertigen - ihres „technischen“ Charakters wegen sind sie öffentlich kaum wahrgenommen worden. Da Grund 2 zudem lediglich im Bericht der WG3, einer „Nicht-Science“-Arbeitsgruppe, kommuniziert wurde, hat dieser Tatbestand die Science-
Kreise erst mit seiner erneuten und deutlich präzisierten Publikation im Synthesis Report (IPCC 2007) erreicht.

Beide Gründe sind einfach zu quantifizieren. Es geht um Relationswerte gegenüber der Diagnose im TAR von 2001 beziehungsweise den zu diesem Zeitpunkt bekannten Fakten. Grund 2 - die Zunahme der (perzipierten) Emissionen - bringt plus 15 Prozent Anstieg. Grund 1 - die Zunahme aufgrund einer korrigierten Einschätzung des langfristigen Effekts eines Anstiegs der THG-Konzentration - bringt etwa plus 20 Prozent. Zusammengenommen ist das (perzipierte) Problem seit Beginn dieses Jahrzehnts um etwa 40 Prozent angestiegen (Details: siehe Luhmann [im Erscheinen]).

Die zentrale Konsequenz: Wir sind der Grenze, dem „gefährlichen Klimawandel“ gemäß Artikel 2 Klimarahmenkonvention (UN Framework Convention on Climate Change, UNFCCC), erheblich näher gekommen - der Abstand ist deutlich geschrumpft, wenn er überhaupt noch besteht. Es fehlt schlagartig an dem Zeitpolster, das bislang für das soziale Lernen, für die gesellschaftliche Diskussion der Klimaherausforderung und zur Konsensbildung einkalkuliert war. Die zweite Konsequenz: Zu ihrer aktuellen Orientierung hinsichtlich der für die Zukunft für noch verfügbar (feasible) zu haltenden Emissionsbudgets muss die Politik sich nach anderen Quellen beziehungsweise Kapazitäten umsehen als allein dem IPCC, dessen Koordinations- und Qualitätssicherungsverfahren sich angesichts der Dringlichkeit der Herausforderung als zu zeitaufwendig herausstellen.

\section{Höher angesetzte Klimasensitivität}

\section{Definition}

Die Folgen, die ein geplantes (oder auch bereits realisiertes) Emissionsbudget erwarten lässt, sind quantitativ zu fassen mittels des Maßes „Klimasensitivität“. Diese gibt an, mit welcher Temperaturerhöhung (in ${ }^{\circ} \mathrm{C}$ ) das Klimasystem auf eine Verdoppelung der Konzentration von (ursprünglich allein) Kohlendio-

2 „Seit dem 3. Sachstandsbericht des IPCC (TAR) 2001 hat die Forschung wesentliche Fortschritte beim Verständnis der aktuellen Klimaänderungen erzielt." (S. 1) sowie „2007 urteilt das IPCC: ,Sehr wahrscheinlich“ seien die anthropogen bedingten THG-Zuwächse die Ursache für die beobachtete globale Temperaturerhöhung in der zweiten Hälfte des 20. Jahrhunderts. Im 3. Sachstandsbericht hieß es noch ,wahrscheinlich،." (S. 2) Aus BMU et al. (2007).

$3 \mathrm{Im}$ TAR hatte das IPCC erstmals in seiner Geschichte auf die Angabe eines best guess verzichtet.

42004 wurden 49 Gigatonnen $\mathrm{CO}_{2}$-Äquivalente pro Jahr ausgestoßen. Seit 1990 sind die Emissionen somit von 39,4 Gigatonnen um knapp 25 Prozent gestiegen, mit deutlich akzelerativer Tendenz in jüngerer Zeit (Zwischenpunkt im Jahr 2000: 44,7 Gigatonnen) (nach IPCC 2007, Fig. SPM.3).

5 Rodung und Drainage moorbasierter Regenwälder in Südostasien haben zu einem Anstieg der (wahrgenommenen) $\mathrm{CO}_{2}$-Emissionen in der Größenordnung der Emissionen der EU-15 geführt.

6 Dieses Szenarienset hat das IPCC 1996 erarbeitet und 2000 abgeschlossen. In ihm konnte ein empirischer Stand der THG-Emissionen höchstens bis 1997/1998 berücksichtigt werden. Erst danach setzte die Akzeleration des globalen Wirtschaftswachstums, insbesondere in Südostasien, ein. 
xid in der Erdatmosphäre in langer Frist reagiert. Sie spielt eine Schlüsselrolle, weil der Klimawandel einen Zeitverzug doppelter Art nach sich zieht: Erstens die jahrzehntelange Verzögerung zwischen dem Setzen der Ursache - der Änderung der atmosphärischen Strahlungsbilanz (gemessen in radiative forcing) - und der Manifestation als Änderung der bodennahen Temperatur; zweitens die jahrhundertelange Verweildauer einmal emittierter anthropogener THG, die unaufhörlich Ursachen setzen. Insbesondere geht es um $\mathrm{CO}_{2}$, aber auch um die fluorierten THG (F-Gase), die treibhauswirksamsten Gase.

Die Hauptdefinition ${ }^{7}$ der Klimasensitivität, equilibrium climate sensitivity, lautet: „(...) equilibrium change in the annual mean global surface temperature following a doubling of the atmospheric equivalent carbon dioxide concentration " (IPCC Working Group I 2007, S. 943). Die Verdoppelung, unabhängig vom Ausgangsniveau, wird als Referenz genommen, weil der Zusammenhang von Ursache und Effekt logarithmisch ist. ${ }^{8}$ Neben $\mathrm{CO}_{2}$ werden weitere langlebige THG emittiert; ihr Anteil ${ }^{9}$ an den aktuellen Emissionen liegt bei gut 20 Prozent. Um diese zu berücksichtigen, wird ergänzend das Konzentrationsmaß „ $\mathrm{CO}_{2}$-eq“ verwendet. Damit lässt sich auch das Konzentrations-Äquivalent eines Temperaturziels angeben (siehe unten).

\section{Bedeutung für Politik und Wirtschaft}

Bedeutung hat das Maß „Klimasensitivität“ in Politik und Wirtschaft. Die Vorgabe eines Ziels gemäß Artikel 2 UNFCCC in Form einer Temperaturobergrenze vermag politisch nicht direkt handlungsleitend zu sein. Handlungsleitend hingegen sind zwei Typen von Angaben, die beide von der Klimasensitivität abhängen. Sie entsprechen zwei Handlungsfeldern: der Mitigationspolitik und den Adaptationsbemühungen.

Mitigationspolitisch zentral und direkt handlungsleitend ist die Setzung einer globalen Emissionsobergrenze, äquivalent das Setzen von Emissionsminderungszielen. Das kann die Politik autonom. Will sie aber Artikel 2 UNFCCC ernst nehmen und wie die EU ${ }^{10} \mathrm{ihr}$ Ziel in eine Wirkungsdimension des Klimawandels umformuliert sehen, ist sie auf klimawissenschaftliche Modelle angewiesen. Basierend auf der geschätzten Klimasensitivität vermögen diese, rückwärts rechnend, aus der Temperaturobergrenze die maximal zulässige THG-Konzentration und daraus wiederum die maximal noch zulässigen Emissionsbudgets zu bestimmen. Das (wirtschaftliche) Korollar dazu sind Mengen (im Wesentlichen) fossiler Energieträger, die „fossil“, das heißt „unter der Erde“, zu bleiben haben, die also ihren Wert verlieren. Solche Abschätzungen entscheiden über (verlustbedrohte) Werte in der Größenordnung fünf- bis sechsstelliger Milliardenbeträge.

Adaptation ist prioritär Sache der potenziell Betroffenen, denn der Klimawandel wirkt unvermittelt und dispers in Raum und Zeit: Es kann jeden treffen, also ist es im ureigensten Interesse eines jeden Eigentümers oder Treuhänders, sich einzurichten auf den Wandel, sei es zum Schutz bestehender Anlagen, sei es in der Auslegung neu zu errichtender Anlagen. „Adaptationspolitik“ ist deshalb sekundär und hat nur eine ertüchtigende und koor-

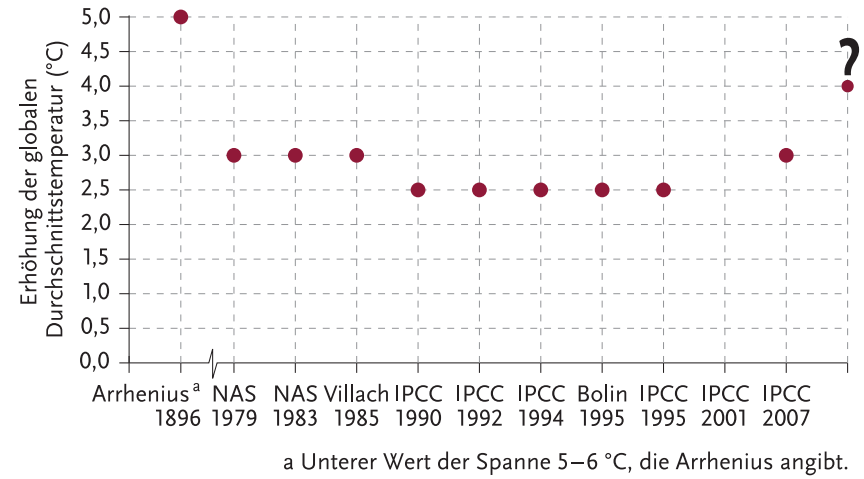

ABBILDUNG 1: „Beste Schätzung“ der Klimasensitivität nach verschiedenen Assessments. Die Klimasensitivität besagt, wie stark eine Verdoppelung der atmosphärischen Treibhausgaskonzentration die globale Durchschnittstemperatur langfristig erhöht. Das IPCC gab 2007 erstmals einen Wert von $3^{\circ} \mathrm{C}$ an und kam damit auf den von der National Academy of Science (NAS) schon 1979 geschätzten Wert zurück. Das IPCC hat also die Gesellschaft während 17 Jahren in falscher Sicherheit gewiegt. Quelle: Van der Sluijs (1997, Kapitel 2); für 1896, 2001 und 2007 ergänzt durch den Autor.

dinierende Rolle - anders als die Mitigationspolitik, die massiv gegen etablierte Gegenwartsinteressen verstoßen muss und deswegen Machtpolitik ist. Im Zentrum der „Adaptationspolitik“ hat das Ausmaß der künftigen Temperaturänderung zu stehen, das aufgrund der bisherigen Emissionen unvermeidlich und also zu erwarten ist - auch das hängt von der Klimasensitivität ab. Die Bezeichnung „real“ vermag diesen Teil zukünftiger Temperaturänderung von demjenigen Teil abzuheben, der nur „prognostiziert" ist, das heißt noch vermieden werden kann.

\section{Quantitative Bestimmung durch das IPCC}

Zu den zentralen Entscheiden der IPCC-Arbeitsgruppe I im AR4 gehört, die „beste Schätzung“ der Klimasensitivität von bisher $2,5^{\circ} \mathrm{C}$ auf $3^{\circ} \mathrm{C}$ angehoben zu haben, also um rund 20 Prozent. ${ }^{11}$ Abbildung 1 zeigt die Geschichte der Bestimmung der „besten Schätzung“. Deutlich wird: Das IPCC geht mit AR4 erstmals in seiner Geschichte auf $3^{\circ} \mathrm{C}$. Es bestätigt somit, dass das Klimasystem doch (mindestens) so sensitiv auf den anthropogenen Treibhausimpuls reagiert, wie die Klimawissenschaft das schon 1979 (National Academy of Science) und Mitte der 1980er Jahre (Villach-Report) diagnostiziert hatte. Das IPCC hatte bei seinem Amts-

7 Nebendefinitionen, die auf leichter handhabbare Ungleichgewichtssituationen bezogen sind, sind die effective climate sensitivity sowie der transient climate response.

8 Das radiative forcing folgt der Konzentration wegen zunehmender Sättigung in den Absorptionsbanden von $\mathrm{CO}_{2}$ degressiv (logarithmisch).

9 Gemeint sind die rechtlich als anthropogen regulierten, also nur die sechs langlebigen THG.

10 ,It confirms that, with a view to achieving the ultimate objective of the UN Framework Convention on Climate Change, the global annual mean surface temperature increase should not exceed $2^{\circ} \mathrm{C}$ above pre-industrial levels" (EU-Ratsbeschluss vom 22./23.03.2005).

11 „The equilibrium climate sensitivity (...) is likely to be in the range 2 to $4.5^{\circ} \mathrm{C}$ with a best estimate of about $3^{\circ} \mathrm{C}$ (...). “ (IPCC Working Group I 2007, S. 12, 65). 


\section{"Gute Wissenschaft" trachtet nach Sicherheit wissenschaftlicher Aussagen; der Gesellschaft hingegen geht es um die reale Sicherheit von Infrastruktur und Vermögen.}

antritt das in den 1970er Jahren erreichte Alarmniveau zurückgenommen und also die Gesellschaft in einer Zwischenperiode von 17 Jahren in falscher Sicherheit gewiegt.

\section{Wann ist der „gefährliche Klimawandel“ erreicht?}

Die Klimasensitivität gibt einen Wert an, der zur rechtlich relevanten, in Artikel 2 UNFCCC vorgegebenen Obergrenze der Temperaturerhöhung in eine einfach zu kommunizierende Beziehung gesetzt werden kann. Dies setzt voraus, dass die Politik Artikel 2 UNFCCC in Form eines langfristigen Grenzwerts des Temperaturanstiegs ausgelegt hat; nur dann ist die Anwendung der equilibrium climate sensitivity angemessen. Mit ihrer Hilfe ist der Abstand zum „gefährlichen“ Bereich mittels einer annähernden Handrechnung leicht zu ermitteln. Man betrachtet allein die sechs langlebigen THG gemäß der bislang leitenden politischen Maxime, die Klimaschutzanstrengungen auf diese zu beschränken. Addiert man die Konzentrationswerte der einzelnen Gase, beläuft sich der Anstieg des radiative forcing heute auf knapp 2,6 Watt pro Quadratmeter gegenüber vorindustrieller Zeit. ${ }^{12}$ Bei einer Klimasensitivität von $3^{\circ} \mathrm{C}$ ist also die Implikation der heutigen THG-Konzentrationsmenge ein Anstieg der Gleichgewichtstemperatur um $2,1^{\circ} \mathrm{C} .{ }^{13}$

Der Wortlaut von Artikel 2 UNFCCC erlaubt jedoch auch andere Auslegungen. Selbst wenn das Ziel als Temperaturziel verstanden ist, muss der „übermäßige“ anthropogene Klimawandel nicht als Überschreiten eines langfristigen Gleichgewichtsgrenzwertes verstanden werden; man kann auch seine Geschwindigkeit als übermäßig ansehen. In diesem Falle ist es legitim, den im EU-Beschluss vom März 2005 erwähnten maximalen Temperaturanstieg von $2^{\circ} \mathrm{C}$ nicht im langfristigen Gleichgewicht, sondern nur bis zum Jahre $2100 \mathrm{zu}$ interpretieren - angesichts der Rechnung im vorstehenden Absatz machen etliche von dieser Interpretationsmöglichkeit Gebrauch.

12 Nach Rahmstorf und Schellnhuber (2006, S. 45) bzw. IPCC Working Group I (2007, Tab. 2.12): 2,63 ( $\pm 0,26) \mathrm{W} / \mathrm{m}^{2}$ für den Anstieg von 1750 bis 2005 , mit einem (ausgewiesenen) Anstieg von 1998 bis 2005 um 0,2 W/m² (!).

13 Dreisatzrechnung; unter Einsetzen der Summe des radiative forcing der bisher emittierten Menge der sechs langlebigen THG $\left(2,6 \mathrm{~W} / \mathrm{m}^{2}\right)$ sowie desjenigen Werts $\left(3,7 \mathrm{~W} / \mathrm{m}^{2}\right)$ des gesamten radiative forcing, der einer Verdoppelung der $\mathrm{CO}_{2}$-Konzentration und damit einer Temperaturzunahme um $3{ }^{\circ} \mathrm{C}$ entspricht: $3,0 \times 2,6 / 3,7=2,1$.
Um interessengebundenen Interpretationen von „Gefahr“ entgegenzuwirken, müssen die in Artikel 2 UNFCCC aufgeführten drei Kriterien, an denen die Gefahr zu bemessen ist, abgeklärt werden. Sie sind sämtlich der wissenschaftlichen Analyse zugänglich. Entsprechende Untersuchungen, die eine Vielzahl von Detailuntersuchungen aggregieren, liegen vor (Hare 2003; vergleiche auch die Literaturliste in Patwardhan et al. 2003). Das IPCC hat im Vorlauf zum AR4 jedoch darauf verzichtet, Artikel 2 UNFCCC in der ansonsten üblichen Art - als Review und Klärung der Positionen, die in der wissenschaftlichen Literatur vorfindlich sind - zu elaborieren, weil es sich bei einer solchen Auslegung um ein "Werturteil“ handle (Patwardhan et al.2003, S. 1). Angesichts dessen, dass die Ökonomiekapitel des AR4 ein Review von Werturteilen enthalten, ist das eine nicht konsistent begründete Entscheidung.

In der Literatur bislang weitgehend unerschlossen ist der Zusammenhang zwischen Stabilität der Klimasensitivität und Auslegung von Artikel 2 UNFCCC. Üblich ist zu unterstellen, dass die Klimasensitivität stabil sei. Auf dieser Annahme beruhen ökonomische Analysen, die bestrebt sind, das „optimale“ Niveau des zuzulassenden anthropogen bedingten Klimawandels zu bestimmen. Gründe für eine solche Stabilitätsannahme sind nicht offensichtlich. Zwar darf die Klimasensitivität sicherlich als konstant in der Nähe der gegenwärtigen Temperatur angesehen werden. Ob auf diese Stabilitätsannahme aber auch in historischer Perspektive noch Verlass ist, ist offen („Runaway-Effekte“). Der Grenzwert nach Artikel 2 UNFCCC kann deshalb legitimerweise auch bestimmt werden als derjenige Punkt, ab dem die Stabilität der Klimasensitivität bei gegebenem Wissen der Klimawissenschaft als nicht mehr gewährleistet anzusehen ist.

\section{Die Science-inhärente Tendenz, die Temperaturerhöhung zu unterschätzen}

„Beste Schätzung“ klingt nach Mittelwert. Beim Schutz des Lebens, gegenüber Risiken mit tödlichem Ausgang in großer Zahl, geben sich Vertreter der Sicherheitskultur normalerweise nicht mit Mittelwerten zufrieden. Man orientiert sich vielmehr an einer Extrembelastung beziehungsweise am unteren Rand der Schwankungsbreite von Materialeigenschaften und fügt einen Sicherheitsaufschlag hinzu. Angesichts dieser professionellen Sitte stellt sich die Frage, weshalb das IPCC eine Überschreitung des Randwerts von $4,5^{\circ} \mathrm{C}$ faktisch ausschließt, indem es diese Option nicht elaboriert. Die Antwort lautet: „Values substantially higher than $4.5^{\circ} \mathrm{C}$ cannot be excluded, but agreement of models with observations is not as good for those values" (IPCC 2007, Summary for policymakers, S. 9; Hervorhebung: H.-J. L.).

Werte für die Klimasensitivität über $4,5^{\circ} \mathrm{C}$ werden also nicht berücksichtigt, weil nach klassischen Science-Maßstäben evaluiert wird und solche Rückkopplungen in der Vergangenheit nicht festgestellt wurden. Es handelt sich bei dem jetzigen Vorgang jedoch um das Aufsatteln einer Warmzeit auf eine Warmzeit, zu der erdhistorisch keine Erfahrungen vorliegen. Um vertrauens- 
würdig zu sein, hat der Parameter Klimasensitivität den zu erwartenden Effekt des auf das Holozän aufsattelnden erdgeschichtlichen Experiments „global flächendeckende Einführung der Industriegesellschaft“ auszudrücken. Die Erstmaligkeit des Vorgangs lässt erwarten, dass positive Rückkopplungen, insbesondere mit der Biosphäre, auftreten, die mit Erfahrungswerten aus der Vergangenheit nicht beschrieben sind. Diese mögliche künftige Andersartigkeit der Klimasensitivität auszuschließen, ist daher nicht wirklich wissenschaftlich.

Ich vermute, dass zukünftig erstmalig auftretende Rückkopplungen - insbesondere aus der Lebenssphäre - zu erwarten sind, so dass die Klimasensitivität nicht als konstant, sondern eher in Abhängigkeit von der Temperaturerhöhung der Atmosphäre sowie der Versauerung der Ozeane ${ }^{14} \mathrm{zu}$ bestimmen ist. Gestützt wird diese Vermutung unter anderem durch einen Blick auf die Art des Erkenntnisfortschritts der Klimawissenschaft hinsichtlich der Klimasensitivität. Der besteht nicht (allein) darin, dass die Sensitivität eines definierten, immer gleichen Klimasystems mit der Zeit genauer bestimmt wird; vielmehr beziehen die Modelle immer weitere, darunter zunehmend nichtphysikalische Kompartimente ein und mutieren von Klima- zu Erdsystemmodellen (Abbildung 2). Auf diesem Fortschrittsweg wird die Bedeutung von „Klimasystem“ erweitert. Anders ausgedrückt: „Klima“ ist kein (feststehender) Begriff der Klimawissenschaft. Durch diese Erweiterung werden Wechselwirkungen und Rückkopplungen insbesondere mit der Lebenssphäre der Erde zunehmend eingebunden und damit erst „anerkannt“.

Änderungen der ausgewiesenen Sensitivität des Klimasystems sind somit die Folge zweier Entwicklungen: Die Modelle werden erstens physikalisch besser und dadurch realistischer, vor allem was die Wasserdampfmodellierung angeht, und sie werden zweitens umfassender bezüglich der berücksichtigten Kompartimente. Was ist als Nettoeffekt dieser beiden Entwicklungen zu erwarten? Zu Ersterer habe ich kein Urteil. Zur zweiten Entwicklung, also zum Prozess der Annäherung des Begriffs „Klimasensitivität“ an den der „Erdsystemsensitivität“, ist meines Erachtens zu erwarten, was vonseiten der Wissenschaft nahegelegt wird: Dass die ausgewiesene Höhe dieses Parameters im Zuge dieses Prozesses weiter ansteigt. Unter den tipping points - das sind bislang in die Modelle nicht integrierte Nichtlinearitätsstellen - scheinen die biogenen von besonderem Ausmaß und Einfluss auf die Sensitivität des Klima-Erdsystems zu sein.
Expertenschätzungen zur Kohlenstoff-Ableitkapazität von Ozeanen (siehe die jüngsten dramatischen Befunde in Le Quéré et al. 2007), zur Stabilität der tropischen Regenwälder (vor allem im Amazonasbecken), zur Labilität von Humusauflagen in Wäldern, zur Methanfreisetzungskapazität sowohl nordischer Tundra als auch aus Methanhydraten in den Ozeanen und zu Moorwäldern in Südostasien (vergleiche Pearce 2007 und Luhmann 2007) lassen vorhersehen, dass die Alarmbotschaft in den kommenden IPCC-Berichten noch gesteigert werden wird. $\mathrm{Zu}$ mal diese Schätzungen in ihrer Wechselwirkung miteinander nicht analysiert sind und in der ausgewiesenen Klimasensitivität nur teilweise gespiegelt werden. ${ }^{15}$

14 Die Ozeane sind die bedeutendere der beiden wesentlichen $\mathrm{CO}_{2}$-Senken im Erdsystem. Nach WBGU (2006) hat sich die „Effizienz“ der Ozeansenke mit steigendem Säuregehalt vermindert, was geochemisch erklärbar ist (Kap. 4.2) Diese Tendenz ist auch in Zukunft zu erwarten.

15 Im AR4 (IPCC Working Group I 2007, Kap. 8.6.1) ist dieses Thema explizit nicht integriert behandelt, sondern durch Verweis auf Kapitel 7 und Kapitel 10 „gelöst“. Am weitestgehenden integriert ist die Darstellung unter Kapitel 8.7.2.5.

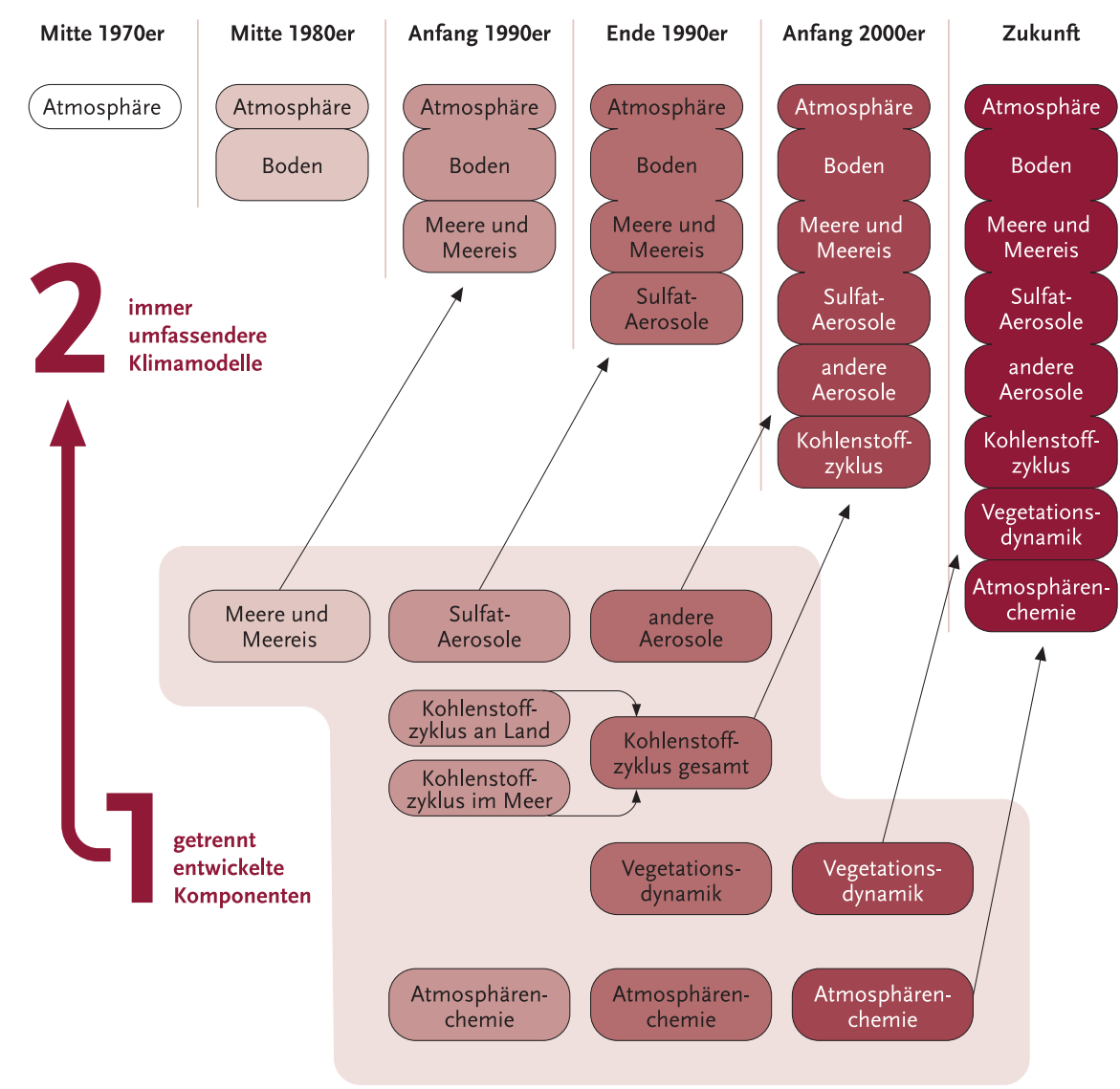

ABBILDUNG 2: Klimamodelle haben sich zu immer umfassenderen (Erdsystem/Klima-) Modellen entwickelt, die immer weitere, auch nichtphysikalische Kompartimente einbeziehen. Annahmen, die der heute prognostizierten Temperaturerhöhung zugrunde liegen, könnten sich daher künftig als unzureichend erweisen. Quelle: IPCC (2001). 


\section{Sicherheit von Aussagen versus Sicherheit von Bevölkerung und Vermögenswerten}

Die konstatierten Engführungen in der Bestimmung der Sensitivität des Klima-Erdsystems sind in der Science-Tradition der Klimawissenschaft angelegt. Die Vertreter(innen) der Klimawissenschaft befinden sich in einem Loyalitätskonflikt gegenüber ihrer Herkunftskultur, der Science-Profession, und gegenüber der Gesellschaft, der zu dienen ihre Ergebnisse gedacht sind. Der Konfliktpunkt trägt den doppelbödigen Namen „Sicherheit“. Die Science-Profession folgt einem Verständnis von "guter Wissenschaft", nach der im Zweifel der Sicherheit ihrer Aussagen der Vorzug zu geben ist. Der Gesellschaft geht es demgegenüber unter "Sicherheit" um die reale Sicherheit von Infrastruktur und Vermögen.

Es besteht somit ein trade-off zwischen dem Gewissheitsanspruch wissenschaftlicher Aussagen und der Sicherheit der potenziell Betroffenen, der zu Warnenden. Dieser Konflikt ist in den Umweltwissenschaften nicht neu, er ist beim Klimathema aber der besonderen Eigenschaften des Gegenstandes wegen zugespitzt. Solange es allein um die Sicherheit von Produziertem - Infrastruktur und technische Anlagen, aber auch Anwendungsprodukte wie Arzneimittel - geht, kann man die Versagenswahrscheinlichkeit des Ganzen mindestens als Summe seiner Teile abschätzen und dem Konflikt hinsichtlich des Restes angemessen entsprechen, indem den Aussagen seitens der Wissenschaft „Sicherheitsaufschläge“ aufgesattelt werden. Letztere haben die Funktion, das von der Wissenschaft sicherlich Ausgeblendete pauschal zu berücksichtigen und auszugleichen. In der Diagnostik des Klimaproblems und somit auch in der Adaptationsplanung fehlt ein äquivalentes Moment. Würde das etablierte sicherheitskulturelle Verhältnis von Wissenschaft und Gesellschaft auch hier zum Tragen kommen, dann wäre es völlig akzeptiert, (mindestens) in der Auslegung von Infrastrukturen und Schutzbauten unter dem Regime des anthropogenen Klimawandels in vergleichbarer Weise vorzugehen.

Diese polarisierte und dysfunktionale Situation bedarf des Ausgleichs. Der Druck zum Ausgleich scheint nicht von der Öffentlichkeitssphäre der Gesellschaft zu kommen, in deren Interesse er liegt - die scheint einstweilen blind für ihre elementarsten Interessen. Als treibende Kräfte kann ich bislang nur zwei innere Spannungen ausmachen. Erstens bei der Wirtschaft, die in ihren Interessen gespalten ist. Bei der Bewertung bestehender Vermögenswerte mag es im Interesse von Eigentümer(inne)n liegen, klimawandelsbedingte reale Abwertungen nicht auszuweisen. Bei der Planung von Neuanlagen hingegen verletzt eine solche Unterlassung die Vermögensinteressen der Investor(inn)en. Diese Spaltung werden die Beteiligten nur begrenzt aushalten.

Eine zweite innere Spannung sehe ich in der Wissenschaft selbst - was für sie spricht. Es geht um den Wandel der Wissenschaft hin zu einer Wissenschaft von der ganzen Natur inklusive des Lebens sowie unter Einbeziehung des Subjekts, also einer "selbstbewussten“ Wissenschaft (vergleiche Ziegler 2008).
Nur eine solche Wissenschaft kann im eigentlichen Sinne „politikberatend" sein: nicht als etwas, was sie zusätzlich tut, sondern inhärent, also perspektivprägend und als gesellschaftliches Subjekt.

\section{Literatur}

BMU et al. 2007. 4. Sachstandsbericht (AR4) des IPCC (2007) über Klimaänderungen: Wissenschaftliche Grundlagen. Kurzzusammenfassung. www.de-ipcc.de/ download/IPCC_WG_I_Kurzfassung.pdf (abgerufen 05.02.2008).

Hare, W. 2003. Assessment of knowledge on impacts of climate change-Contribution to the specification of Art. 2 of the UNFCCC. Materialien. Berlin: WBGU. www.wbgu.de/wbgu_sn2003_ex01.pdf (abgerufen 01.02.2008)

Heisenberg, W. 1943/1944. Die Ordnung der Wirklichkeit. Unveröffentlicht.

IPCC. 2001. Climate change 2001. www.ipcc.ch/ipccreports/tar (abgerufen 08.02.2008).

IPCC. 2007. Climate change 2007: Synthesis report. www.ipcc.ch/ipccreports/ar4-syr.htm (abgerufen 06.02.2008).

IPCC Working Group I. 2007. Climate change 2007: The physical science basis. www.ipcc.ch/ipccreports/ar4-wg1.htm (abgerufen 06.02.2008).

IPCC Working Group II. 2007. Climate change 2007: Impact, adaptation and vulnerability. www.ipcc.ch/ipccreports/ar4-wg2.htm (abgerufen 06.02.2008)

IPCC Working Group III. 2007. Climate change 2007: Mitigation of climate change. www.ipcc.ch/ipccreports/ar4-wg3.htm (abgerufen 06.02.2008).

Le Quéré, C. et al. 2007. Saturation of the Southern ocean $\mathrm{CO}_{2}$ sink due to recent climate change. Science 316: 1735-1738.

Luhmann, H.-J. 2007. Klimapolitik auf „dünner Erdenhaut“. Editorial. GAIA 16/2: 81.

Luhmann, H.-J. Im Erscheinen. Das Klimathema läuft auf eine Klimax zu. Das Ziel multilateraler Klimapolitik nach den IPCC-Berichten des Frühjahrs 2007 und dem G8-Gipfel in Heiligendamm. Technikfolgenabschätzung Theorie und Praxis 16/1.

Patwardhan, A., S. H. Schneider, S. M. Semenov. 2003. Assessing the science to address UNFCCC Article 2. IPCC concept paper. www.ipcc.ch/activity/cct3.pdf (abgerufen 01.02.2008).

Pearce, F. 2007. Das Wetter von morgen: Wenn das Klima zur Bedrohung wird. München: Kunstmann.

Rahmstorf, S., H.J. Schellnhuber. 2006. Der Klimawandel: Diagnose, Prognose, Therapie. München: C.H. Beck.

Van der Sluijs, J. P. 1997. Anchoring amid uncertainty. On the management of uncertainties in risk assessment of anthropogenic climate change. Dissertation, Universität Utrecht.

WBGU (Wissenschaftlicher Beirat der Bundesregierung Globale Umweltveränderungen). 2006. Die Zukunft der Meere - zu warm, zu hoch, zu sauer. Sondergutachten 2006. Berlin: WBGU.

Ziegler, H. 2008. Adaptation versus mitigation - Zur Begriffspolitik in der Klimadebatte. GAIA 17/1: 19-24.

Eingegangen am 25. Juni 2007; überarbeitete Fassung angenommen am 21. Januar 2008.

Hans-Jochen Luhmann

Geboren 1946 in Hamburg. 1968 bis 1973 Studium der Mathematik, Volkswirtschaftslehre und Philosophie. Nach Tätigkeit in der Industrie seit 1994 am Wuppertal Institut für Klima, Umwelt, Energie, gegenwärtig als Projektleiter in der Forschungsgruppe „Zukünftige Energieund Mobilitätsstrukturen“. Herausgeber von GAIA und

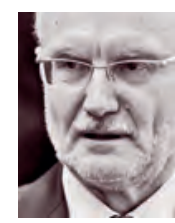

Chefredakteur des Wuppertal Bulletins zu Instrumenten des Klima- und Umweltschutzes. Beiratsmitglied der Vereinigung Deutscher Wissenschaftler. Tätig in den Feldern Klima- und Energiepolitik, Risikopolitik und Wissenschaftspolitik. 